\title{
MASSA SECA E ACÚMULO DE NUTRIENTES EM MUDAS ENXERTADAS DE PEREIRA EM SISTEMA HIDROPÔNICO ${ }^{1}$
}

\author{
ALINE DAS GRAÇAS SOUZA², NILTON NAGIB JORGE CHALFUN ${ }^{3}$, VALDEMAR FAQUIN ${ }^{4}$, \\ ADEMÁRIA APARECIDA DE SOUZA ${ }^{5}$, ANTÔNIO LUCRÉCIO DOS SANTOS NETO ${ }^{6}$
}

RESUMO - O trabalho objetivou determinar a produção de massa da matéria seca das partes da planta e o acúmulo total de macro e micronutrientes pelas mudas enxertadas de cultivares de pereira em condições hidropônicas. Utilizou-se do delineamento experimental inteiramente casualizado, em esquema fatorial 3 x 3, com quatro repetições. Os fatores em estudo foram: três cultivares (Triunfo, Tenra e Cascatense) e três tipos de enxertia (borbulhia em placa - BP; borbulhia em ' $\mathrm{T}$ ' invertido - BT, e garfagem de fenda cheia GF). Avaliaram-se a massa seca da parte área, do sistema radicular e total, e o acúmulo total de nutrientes. O método de enxertia de garfagem de fenda cheia e as cultivares Tenra e Triunfo são indicados para a produção de mudas de pereira em sistema hidropônico. No acúmulo de nutrientes nas mudas de pereira, independentemente da cultivar, estabeleceu-se a seguinte ordem para macronutrientes: $\mathrm{N}>\mathrm{Mg}>\mathrm{K}>\mathrm{Ca}>\mathrm{P}>\mathrm{S}$; e para os micronutrientes: $\mathrm{Fe}>\mathrm{Mn}>\mathrm{B}>\mathrm{Zn}>\mathrm{Cu}$.

Termos para indexação: Pyrus sp., sistema de cultivo sem solo, propagação vegetativa, cultivares.

\section{DRY MATTER AND NUTRIENT ACCUMULATION IN GRAFTED PEAR SEEDLINGS IN HYDROPONIC SYSTEM}

\begin{abstract}
The production of grafted seedlings of pear and peach trees in hydroponics is recent and has been becoming a promising propagation method with a significant reduction in the seedling production time. This study was conducted in order to determine the dry matter of plant parts and the accumulation of macro and micronutrients by pear grafted seedlings in hydroponic conditions. It was used a completely randomized factorial scheme $3 \times 3$ with four replications was. Each replication was made up of two plants (one in each tube), amounting to 72 plants. The factors under study were: three cultivars (Triunfo, Tenra and Cascatense) and three sorts of grafting (bench grafting - BP, inverted T budding -BT and cleft grafting -GF). The nutrient accumulation in pear seedlings, regardless of the cultivar, settled in the following order for macronutrients: $\mathrm{N}>\mathrm{Mg}>\mathrm{K}>\mathrm{Ca}>\mathrm{P}>\mathrm{S}$; and micronutrients: $\mathrm{Fe}>\mathrm{Mn}>\mathrm{B}>\mathrm{Zn}>\mathrm{Cu}$.
\end{abstract}

Index terms: Pyrus sp., soilless growing system, vegetative propagation, cultivate.

\footnotetext{
'(Trabalho 020-14). Recebido em: 16-01-2014. Aceito para publicação em: 08-12-2014.

${ }^{2}$ Pós-Doutoranda em Fitotecnia, UFLA - Campus Universitário, Lavras-MG. E-mail: alinedasgracas@yahoo.com.br

${ }^{3}$ Professor Titular do Departamento de Ciência do Solo,UFLA - Campus Universitário, Lavras-MG. E-mail: vafaquin@dcs.ufla.br ${ }^{4}$ Professor Titular do Departamento de Fitotecnia ,UFLA - Campus Universitário, Lavras-MG. E-mail: nchalfun@dag.ufla.br ${ }^{5}$ Professora do Departamento de Ciências Exatas da Universidade Federal de Alagoas,UFAL - Campus Universitário, Arapiraca-AL. E-mail: ademariasouza@yahoo.com.br

${ }^{6}$ Professor do Departamento de Agronomia, UFAL - Campus Universitário, Arapiraca-AL. E-mail: santosneto@gmail.com
} 


\section{INTRODUÇÃO}

Na fruticultura moderna, têm-se buscado cada vez mais tecnologias que possibilitem a produção de frutas de alta qualidade com menor investimento e alto retorno econômico. Atualmente, existe um grande interesse em pesquisas envolvendo produção de mudas de pereiras, representando uma grande oportunidade de minimizar as importações de mudas. Segundo Fachinello et al. (2008), a muda pode ser considerada como o alicerce da fruticultura, pois dela depende o sucesso ou o fracasso da implantação de um pomar.

Nesse sentido, o aumento na disponibilidade de mudas de qualidade e de menor custo aos fruticultores é essencial para a ampliação de pomares e, consequentemente, para o aumento da produção de frutas no Brasil (FACHINELLO et al., 2008).

É comum na produção de mudas de frutíferas realizar o processo de enxertia, uma vez que as plantas provenientes de sementes apresentam variações devido à segregação genética. Porém existem vários métodos de enxertia que podem ser utilizados na produção de mudas de pereira, como a garfagem e a borbulhia (PASQUAL et al., 2001; HARTMANN et al., 2002). Entretanto, não existem pesquisas que indiquem o melhor procedimento de enxertia na produção de mudas de pereira em sistema hidropônico.

Além dos métodos de enxertia, a utilização de diferentes cultivares de pereira pode influenciar nos resultados para a obtenção de mudas de qualidade. É comum os produtores de pereira utilizarem na produção comercial várias cultivares, dentre as quais se podem destacar a 'Cascatense', 'Tenra' e a 'Triunfo', uma vez que são bem adaptadas às condições de inverno brando do Estado de Minas Gerais. Também não se conhece a resposta dessas cultivares quando se utiliza o sistema hidropônico para a produção de mudas.

A produção convencional de mudas de pereira vem sendo feita em recipientes, que têm como característica a utilização de grandes volumes de substrato, bem como longo período de formação das mudas enxertadas, aproximadamente 450 dias (BARBOSA et al., 1995), resultando em custos elevados de produção. Buscando reduzir o tempo e o custo de produção das mudas de pereira, o sistema hidropônico é uma alternativa viável para os viveiristas, uma vez que é possível produzir a muda quatro vezes mais rápido (SOUZA et al., 2011a) do que aquela obtida no sistema tradicional (BARBOSA et al., 1995).

Dentre os novos métodos para o aumento da produção de mudas de alta qualidade, o cultivo hidropônico tem sido utilizado para várias culturas. Além da produção de hortaliças de folhas, citam-se espécies florestais, ornamentais, fumo e frutíferas (FURLANI, 1999; ROZANE et al., 2011; FREITAS et al., 2011; RADIN et al., 2011; SOUZA et al., 2011a; SOUZA et al., 2011b; SOUZA et al., 2013).

A técnica de hidroponia exige o fornecimento dos nutrientes necessários ao crescimento das plantas de forma adequada e constante, para que se obtenha um bom crescimento vegetal. A produção de mudas enxertadas de pereira em hidroponia é um método promissor (SOUZA et al., 2011b), e a utilização de mudas de pereira com adequado estado nutricional, sadias e vigorosas, é um fator que determina o sucesso da implantação de um pomar (SOUZA, 2010) . Assim, estudos relacionados à nutrição de mudas produzidas nesse sistema tornam-se imprescindíveis na implantação de novos pomares de pereira.

O presente trabalho objetivou determinar a produção de massa seca das partes da muda e o acúmulo total de nutrientes em mudas enxertadas de cultivares de pereira em sistema hidropônico.

\section{MATERIAL E MÉTODOS}

O presente trabalho foi conduzido no Setor de Hidroponia e no Laboratório de Nutrição Mineral de Plantas, do Departamento de Ciência do Solo, da Universidade Federal de Lavras (UFLA). O delineamento experimental utilizado foi $\mathrm{o}$ inteiramente casualizado, em esquema fatorial $3 \mathrm{x}$ 3 , com quatro repetições. Cada parcela foi composta por quatro plantas. Os fatores em estudo foram: três cultivares copa ('Triunfo', 'Tenra' e 'Cascatense') e três tipos de enxertia (borbulhia em placa (BP); borbulhia em T invertido (BT), e garfagem de fenda cheia (GF)).

A cultivar utilizada para o porta-enxerto de pereira foi a 'Taiwan Naschi-C', originária de plantas-matrizes do Instituto Agronômico de Campinas (IAC). Após o período de estratificação, as sementes pré-germinadas desse porta-enxerto de pereira foram selecionadas e semeadas em tubetes plásticos, com $5 \mathrm{~cm}$ de diâmetro e $20 \mathrm{~cm}$ de altura, contendo como substrato a vermiculita. Os tubetes permaneceram em três caixas rasas niveladas, onde passaram a receber solução nutritiva proposta por Faquin e Chalfun (2008). Quando o portaenxerto atingiu cerca de 4-6 mm de diâmetro do caule, iniciou-se a segunda etapa do experimento, enxertando-se as pereiras das cultivares mais bem adaptadas às condições de inverno brando do Estado 
de Minas Gerais ('Cascatense', 'Tenra’ e 'Triunfo').

Os enxertos das três cultivares de pereira foram obtidos de ramos com 4-6 mm de diâmetro do caule, provenientes do IAC, para a realização dos três métodos de enxertia (borbulhia em placa, borbulhia em ' $\mathrm{T}$ ' invertido e garfagem de fenda cheia). A enxertia foi realizada a $15 \mathrm{~cm}$ do colo do porta-enxerto, no período da manhã, utilizando-se de fita plástica transparente, para permitir melhor aderência do garfo e da borbulha. As mudas enxertadas permaneceram em caixas rasas niveladas, previamente dimensionadas, com tamanho suficiente para comportar os tubetes e ligadas a um reservatório de 1.000 litros de solução nutritiva.

$\mathrm{Na}$ caixa rasa nivelada, os tubetes permaneceram com a parte inferior imersa a uma lâmina de cerca de $5,0 \mathrm{~cm}$ de solução nutritiva. Assim, a irrigação e a nutrição das plantas foram realizadas por capilaridade da própria vermiculita. A circulação da solução nutritiva nas caixas rasas foi realizada por meio de uma motobomba ligada ao reservatório. A reposição de nutrientes na solução nutritiva do reservatório foi efetuada por meio da condutividade elétrica, ajustando-se diariamente seu valor para $1,6 \mathrm{~ms} \mathrm{~cm}^{-1}$, pela adição de soluçõesestoque de macro e micronutrientes, preparadas de acordo com Faquin e Chalfun (2008).

Aos 112 dias após a semeadura, quando as mudas de pereira estavam aptas a serem plantadas em campo, de acordo com os critérios de altura $(50 \mathrm{~cm})$ e diâmetro $(0,5 \mathrm{~cm})$ estabelecidos pela Portaria $\mathrm{n}^{\mathrm{o}} 37$ -Anexo IX, as plantas foram coletadas e separadas. O tempo de produção das mudas foi de quatro meses. As raízes foram cuidadosamente lavadas em água corrente, para a eliminação de resíduos de vermiculita e nutrientes da solução nutritiva. Posteriormente, foram secas em estufa de circulação forçada de ar, a 60- $65^{\circ} \mathrm{C}$, até massa constante e, em seguida, pesadas em balança semianalítica.

A massa seca das partes das plantas foi moída em moinho tipo Willey, com malha de 20 mesh, e os teores macro (N, P, K, Ca, Mg e S) e micronutrientes ( $\mathrm{B}, \mathrm{Cu}, \mathrm{Fe}, \mathrm{Mn}$ e $\mathrm{Zn}$ ) foram analisados (MALAVOLTA et al., 1997).

A produção de massa seca das diferentes partes e a total foram analisadas separadamente. A determinação da quantidade de nutrientes acumulados foi obtida pelo produto entre os teores dos nutrientes e a massa seca da parte referida, e a do acúmulo total dos nutrientes foi pela soma dos acúmulos em cada parte da planta.

As variáveis avaliadas foram submetidas à análise estatística pelo programa estatístico Sisvar (FERREIRA, 2011), realizando-se a análise de variância e o teste de Tukey, a 5\% de probabilidade, para comparação de médias.

\section{RESULTADOS E DISCUSSÃO}

A massa seca da raiz, da parte aérea e do total das mudas de pereira foi influenciada de forma significativa pelas cultivares e tipos de enxertia, não sendo observada interação entre os fatores (Figuras $1 \mathrm{~A}$ e B).

A maior produção de matéria seca da raiz, da parte aérea e do total das mudas foi obtida com o método de enxertia de garfagem de fenda cheia (Figura 1A), com valores de 6,0 g, 18,0 g e $24,0 \mathrm{~g}$, respectivamente. Esse resultado pode ser atribuído à maior velocidade de pegamento devido à melhor justaposição dos tecidos do enxerto e do porta- enxerto das mudas de pereira pelo método de garfagem em fenda cheia (SOUZA et al., 2011a).

Em relação às cultivares, a Triunfo e a Tenra (Figura 1B) proporcionaram maior acúmulo de massa seca da raiz, da parte aérea e do total. Para a cultivar Triunfo, a produção da massa seca da raiz, da parte aérea e do total foi de 5,0 g, 15,5 g e 20,5 $\mathrm{g}$, respectivamente. Em relação à cultivar Tenra, a produção da massa seca da raiz, da parte aérea e do total foi de 4,5 g, 14,5 g e 19,0 g, respectivamente.

Segundo Echer et al. (2006), a massa seca do sistema radicular e da parte aérea indica a rusticidade de uma muda. Quanto maior a massa, mais rústica será. Tomando como base esta afirmação, as mudas obtidas do método de garfagem de fenda cheia com as cultivares Triunfo e Tenra podem ser consideradas mais rústicas que as demais.

Pesquisas realizadas por Seifert et al. (2009), avaliando a viabilidade do uso de porta-enxerto de marmeleiro 'Japonês' enxertado com as cultivares das pereiras 'Triufo' e 'Seleta', nos métodos de borbulhia e garfagem, obtiveram maior acúmulo de massa seca da parte aérea com a enxertia de garfagem de fenda cheia e o uso da cultivar Seleta.

Em trabalho realizado por Mendes (2007), avaliando diferentes tratamentos de poda do portaenxerto 'Okinawa' e diferentes métodos de enxertia na formação de mudas de pessegueiro da cultivar Premier, em condições hidropônicas, ele obteve maiores valores da produção de massa seca da raiz, da parte aérea e do total com a enxertia de borbulhia em placa e borbulhia em ' $\mathrm{T}$ ' invertido, no tratamento sem poda e no método de garfagem de fenda cheia com poda. No presente estudo, resultados semelhantes foram obtidos com métodos de garfagem de fenda cheia com poda.

O acúmulo de nutrientes nas mudas, de 
maneira geral, apresentou resultados semelhantes àqueles obtidos na produção de massa seca. Os valores médios dos acúmulos totais de macro e micronutrientes nas mudas de pereiras são apresentados nas Tabelas 1; 2; 3; 4 e 5 .

As cultivares Tenra e Triunfo, e o método de enxertia de garfagem de fenda cheia produziram maior quantidade de massa seca e promoveram, também, maior acúmulo dos nutrientes nas mudas de pereira (Tabelas 1 e 2; Figuras 1A e 1B).

Comparando-se os métodos de enxertia, observa-se que o maior acúmulo total de macronutrientes (Tabela 3) e de micronutrientes (Tabela 4) nas mudas de pereira foi obtido no método garfagem de fenda cheia.

Para as mudas de pereira avaliadas, obtevese a seguinte ordem para macronutrientes: $\mathrm{N}>$ $\mathrm{Mg}>\mathrm{K}>\mathrm{Ca}>\mathrm{P}>\mathrm{S}$ e para os micronutrientes: $\mathrm{Fe}>\mathrm{Mn}>\mathrm{B}>\mathrm{Zn}>\mathrm{Cu}$ (Tabela 5). Os resultados apresentados no presente trabalho podem ser utilizados como referenciais das exigências das mudas das cultivares de pereira Cascatense, Tenra e Triunfo, comercialmente prontas em sistema hidropônico. Trabalho realizado por Mendes (2007), utilizando o porta-enxerto 'Okinawa' enxertado com a cultivar Premier, também em condições hidropônicas, obteve a mesma ordem decrescente dos resultados obtidos no presente estudo para macro e micronutrientes $>\mathrm{Mg}>\mathrm{K}>\mathrm{Ca}>\mathrm{P}>\mathrm{S}$ e $\mathrm{Fe}>\mathrm{Mn}>\mathrm{B}>\mathrm{Zn}>\mathrm{Cu}$.
Resultados semelhantes foram encontrados por Souza (2010), utilizando a mesma solução nutritiva do presente estudo; entretanto, para a obtenção de mudas de pessegueiro, foi utilizada a seguinte ordem decrescente dos macronutrientes: $\mathrm{N}>\mathrm{Mg}$ $>\mathrm{K}>\mathrm{Ca}>\mathrm{P}>\mathrm{S}$; e micronutrientes: $\mathrm{Fe}>\mathrm{Mn}>\mathrm{B}>\mathrm{Zn}>\mathrm{Cu}$.

Corroborando os dados obtidos neste trabalho, Martins et al. (2013), trabalhando com porta-enxertos de Psidium guajava, utilizando a mesma solução nutritiva do presente estudo, obtiveram a mesma ordem decrescente para macro e micronutrientes.

Trabalhos com produção de massa seca e acúmulo de nutrientes para as espécies do gênero Pyrus são escassos. Pesquisas têm enfatizado somente teor foliar de macronutrientes e micronutrientes (VERÍSSIMO et al., 2006; KNAPIK;ANGELO, 2007; MARCANTE et al., 2010) e absorção foliar com boro (PICCHIONI et al., 1995). Assim, a intensificação dos estudos de produção de massa seca e acúmulo de nutrientes das espécies deste gênero pode contribuir muito para a cultura da pereira.

Verificou-se que as mudas de pereira cresceram e desenvolveram-se adequadamente em sistema de produção hidropônica, uma vez que não apresentaram sintomas de deficiência ou excesso nutricional. De acordo com Rodrigues (2002), a produção hidropônica diminui o tempo de formação e reduz o custo de produção de mudas de qualidade.

TABELA 1 - Acúmulo total de macronutrientes $\left(\mathrm{mg} \mathrm{planta}^{-1}\right)$ em mudas de pereira de diferentes cultivares, mantidas em hidroponia.

\begin{tabular}{ccccccc}
\hline Cultivares & $\mathbf{N}$ & $\mathbf{P}$ & $\mathbf{K}$ & $\mathbf{C a}$ & $\mathbf{M g}$ & $\mathbf{S}$ \\
\hline Tenra & $536,25 \mathrm{a}^{(1)}$ & $51,33 \mathrm{a}$ & $268,12 \mathrm{a}$ & $102,85 \mathrm{a}$ & $374,12 \mathrm{~b}$ & $15,21 \mathrm{a}$ \\
Triunfo & $575,25 \mathrm{a}$ & $47,67 \mathrm{a}$ & $287,62 \mathrm{a}$ & $108,66 \mathrm{a}$ & $392,06 \mathrm{a}$ & $15,89 \mathrm{a}$ \\
Cascatense & $452,25 \mathrm{~b}$ & $40,22 \mathrm{~b}$ & $226,12 \mathrm{~b}$ & $86,84 \mathrm{~b}$ & $314,16 \mathrm{~b}$ & $12,77 \mathrm{~b}$ \\
\hline $\mathbf{C V}$ & 18,54 & 18,01 & 18,55 & 16,66 & 16,53 & 16,45 \\
\hline
\end{tabular}

${ }^{(1)}$ Médias seguidas pela mesma letra na coluna não diferem entre si, a 5\%, pelo teste de Tukey.

TABELA 2 - Acúmulo total de micronutrientes $\left(\mu \mathrm{g}_{\mathrm{glanta}}{ }^{-1}\right)$ de mudas de pereira de diferentes cultivares, mantidas em hidroponia.

\begin{tabular}{lccccc}
\hline \multicolumn{1}{c}{ Cultivares } & $\mathbf{B}$ & $\mathbf{C u}$ & $\mathbf{F e}$ & $\mathbf{M n}$ & $\mathbf{Z n}$ \\
\hline Tenra & $733,42 \mathrm{a}^{(1)}$ & $59,58 \mathrm{a}$ & $3.453,25 \mathrm{a}$ & $786,33 \mathrm{a}$ & $369,42 \mathrm{a}$ \\
Triunfo & $806,00 \mathrm{a}$ & $64,25 \mathrm{a}$ & $3.633,75 \mathrm{a}$ & $868,00 \mathrm{a}$ & $396,28 \mathrm{a}$ \\
Cascatense & $609,92 \mathrm{~b}$ & $50,250 \mathrm{~b}$ & $2.945,00 \mathrm{~b}$ & $656,83 \mathrm{~b}$ & $313,10 \mathrm{~b}$ \\
\hline CV & 19,26 & 18,56 & 17,69 & 19,09 & 18,33 \\
\hline
\end{tabular}

${ }^{(1)}$ Médias seguidas pela mesma letra na coluna não diferem entre si, a $5 \%$, pelo teste de Tukey. 
TABELA 3 - Acúmulo total de macronutrientes $\left(\mathrm{mg}\right.$ planta $\left.^{-1}\right)$ em mudas de pereira de diferentes métodos de enxertia, mantidas em hidroponia.

\begin{tabular}{ccccccc}
\hline Método de enxertia & $\mathbf{N}$ & $\mathbf{P}$ & $\mathbf{K}$ & $\mathbf{C a}$ & $\mathbf{M g}$ & $\mathbf{S}$ \\
\hline Borbulhia em placa & $461,25 \mathrm{~b}^{(1)}$ & $41,20 \mathrm{~b}$ & $230,62 \mathrm{~b}$ & $88,40 \mathrm{~b}$ & $318,77 \mathrm{~b}$ & $12,92 \mathrm{~b}$ \\
Borbulhia T invertido & $405,75 \mathrm{~b}$ & $36,07 \mathrm{~b}$ & $202,87 \mathrm{~b}$ & $77,49 \mathrm{~b}$ & $282,39 \mathrm{~b}$ & $11,48 \mathrm{~b}$ \\
Garfagem fenda cheia & $696,75 \mathrm{a}$ & $61,95 \mathrm{a}$ & $348,37 \mathrm{a}$ & $132,46 \mathrm{a}$ & $479,19 \mathrm{a}$ & $19,48 \mathrm{a}$ \\
\hline CV \% & 18,54 & 18,01 & 18,55 & 16,66 & 16,53 & 16,45
\end{tabular}

${ }^{(1)}$ Médias seguidas pela mesma letra na coluna não diferem entre si, a 5\%, pelo teste de Tukey.

TABELA 4 - Acúmulo total de micronutrientes $\left(\mu \mathrm{g} \mathrm{planta}^{-1}\right)$ de mudas de pereira em diferentes métodos de enxertia, mantidas em hidroponia.

\begin{tabular}{cccccc}
\hline Método de enxertia & $\mathbf{B}$ & $\mathbf{C u}$ & $\mathbf{F e}$ & $\mathbf{M n}$ & $\mathbf{Z n}$ \\
\hline Borbulhia em placa & $622,92 \mathrm{~b}^{(1)}$ & $51,25 \mathrm{~b}$ & $2.954,50 \mathrm{~b}$ & $670,83 \mathrm{~b}$ & $317,75 \mathrm{~b}$ \\
Borbulhia T invertido & $525,42 \mathrm{~b}$ & $45,58 \mathrm{~b}$ & $2.631,50 \mathrm{~b}$ & $565,83 \mathrm{~b}$ & $282,10 \mathrm{~b}$ \\
Garfagem fenda cheia & $1.001,00 \mathrm{a}$ & $77,25 \mathrm{a}$ & $4.446,00 \mathrm{a}$ & $1.074,50 \mathrm{a}$ & $478,95 \mathrm{a}$ \\
\hline $\mathbf{C V} \%$ & 19,26 & 18,56 & 17,69 & 19,09 & 18,33 \\
\hline
\end{tabular}

${ }^{(1)}$ Médias seguidas pela mesma letra na coluna não diferem entre si, a 5\%, pelo teste de Tukey.

TABELA 5- Acúmulo total de macro (mg planta $\left.{ }^{-1}\right)$ e micronutrientes $\left(\mu\right.$ planta $\left.^{-1}\right)$, em ordem decrescente, em mudas de cultivares de pereira, mantidas em hidroponia.

\begin{tabular}{lll}
\hline \multicolumn{1}{c}{ Cultivares } & \multicolumn{1}{c}{ Macronutrientes } & Micronutrientes \\
\hline Tenra & $\mathrm{N}>\mathrm{Mg}>\mathrm{K}>\mathrm{Ca}>\mathrm{P}>\mathrm{S}$ & $\mathrm{Fe}>\mathrm{Mn}>\mathrm{B}>\mathrm{Zn}>\mathrm{Cu}$ \\
Triunfo & $\mathrm{N}>\mathrm{Mg}>\mathrm{K}>\mathrm{Ca}>\mathrm{P}>\mathrm{S}$ & $\mathrm{Fe}>\mathrm{Mn}>\mathrm{B}>\mathrm{Zn}>\mathrm{Cu}$ \\
Cascatense & $\mathrm{N}>\mathrm{Mg}>\mathrm{K}>\mathrm{Ca}>\mathrm{P}>\mathrm{S}$ & $\mathrm{Fe}>\mathrm{Mn}>\mathrm{B}>\mathrm{Zn}>\mathrm{Cu}$ \\
\hline
\end{tabular}



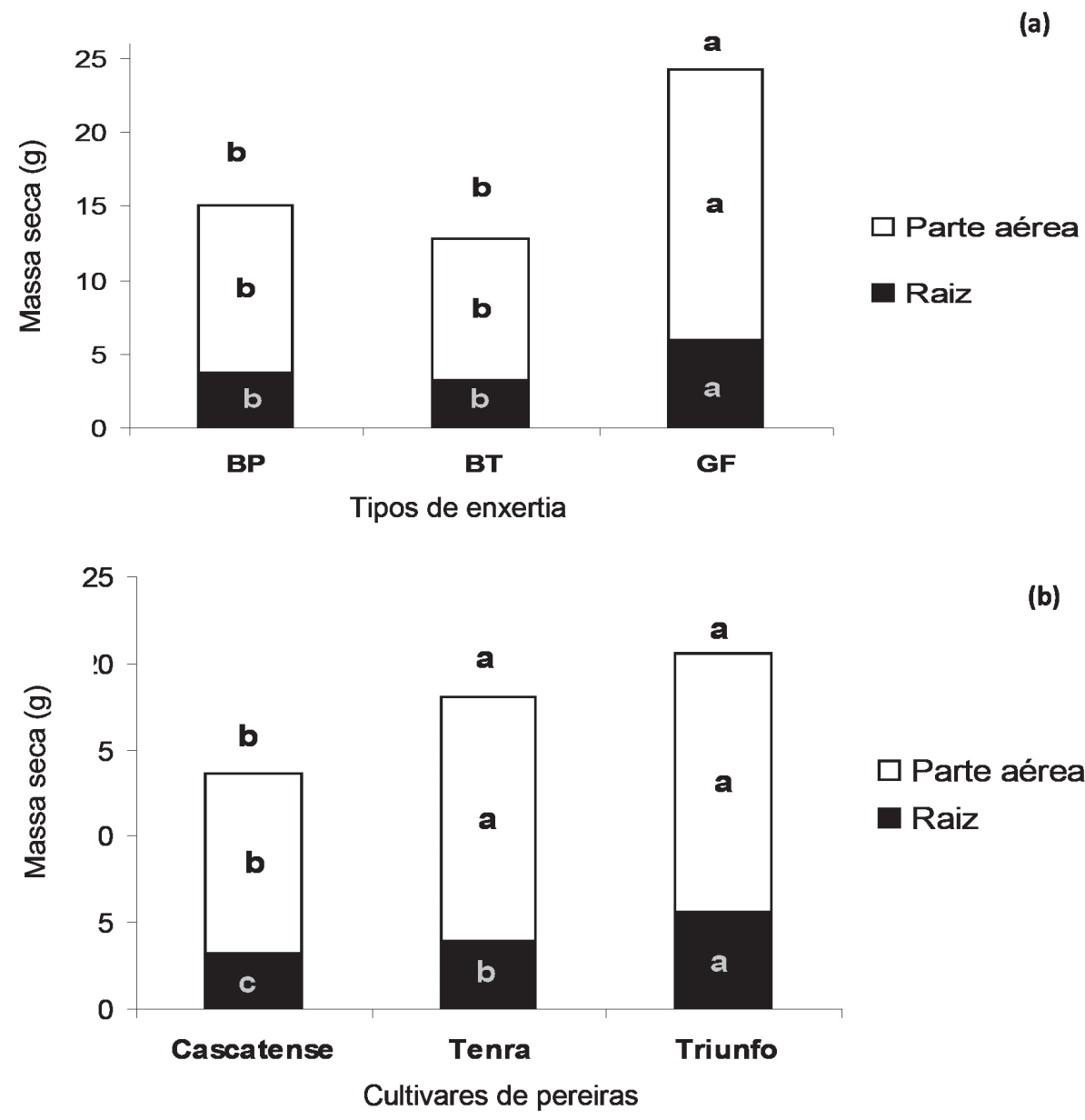

FIGURA 1- Produção de matéria seca da raiz, da parte aérea e total em função dos métodos de enxertia em mudas de pereira (BP: borbulhia em placa; BT: borbulhia em 'T' invertido; GF: garfagem de fenda cheia) - (A) e cultivares de pereira 'Cascatense', 'Tenra' e 'Triunfo' (B). Para cada parte e total, médias seguidas pela mesma letra não diferem entre si, a 5\%, pelo teste de Tukey.

\section{CONCLUSÃO}

O método de enxertia de garfagem de fenda cheia e as cultivares Tenra e Triunfo são indicados para a produção de mudas de pereira em sistema hidropônico.

No acúmulo de nutrientes nas mudas de pereira, independentemente da cultivar, estabeleceuse a seguinte ordem para macronutrientes: $\mathrm{N}>\mathrm{Mg}>\mathrm{K}>\mathrm{Ca}>\mathrm{P}>\mathrm{S}$; e para os micronutrientes: $\mathrm{Fe}>\mathrm{Mn}>\mathrm{B}>\mathrm{Zn}>\mathrm{Cu}$.

\section{REFERÊNCIAS}

ECHER, M. M.; GUIMARÃES, V. F.; KRIESER, C. R.; ABUCARMA, V. M.; KLEIN, J.; SANTOS, L.; DALLABRIDA, W. R. Uso de bioestimulante na formação de mudas de maracujazeiro-amarelo. Semina: Ciência Agrária, Londrina, v. 27, n. 3, p. 351-360, 2006.

FACHINELLO, J. C.; NACHTIGAL, J. C.; KERSTEN, E. Fruticultura: fundamentos e práticas. Pelotas: Editora UFPEL, 2008. 176 p.

FAQUIN, V.; CHALFUN, N. N. J. Hidromudas: processo de produção de porta-enxerto de mudas frutíferas, florestais e ornamentais enxertadas em hidroponia (BRN. PI 0802792-7). Rio de Janeiro: INPI, 2008. 
FERREIRA, D. F. Sisvar: a computer statistical analysis system. Ciência e Agrotecnologia, Lavras, v. 35, n. 6, p. 1039-1042, 2011.

FURLANI, P. R. Hydroponic vegetable production in Brazil. Acta Horticulturae, The Hague, v. 481, p. $777-778,1999$.

HARTMANN, H.T.; KESTER, D. E.; DAVIES JÚNIOR, F. T.; GENEVE, R. L. Plant propagation: principles and practies. $7^{\text {th }}$ ed. New Jersey: Prentice Hall, 2002. 880p.

KNAPIK, J. G.; ANGELO, A. C. Crescimento de mudas de Prunus sellowii koehne em resposta a adubações com NPK e pó de basalto. Floresta, Curitiba, v. 37, n. 2, p. 257-264, 2007.

MALAVOLTA, E.; VITTI, G.C.; OLIVEIRA, S.A. Avaliação do estado nutricional de plantas: princípios e aplicações. 2.ed. Piracicaba: Potafós, $1997.319 \mathrm{p}$.

MARCANTE, N. C.; PRADO, R. M.; CAMACHO, M. A.; ROSSET, J. S.; ECCO, M.; SAVAN, P. A. L. Determinação da matéria seca e teores de macronutrientes em folhas de frutíferas usando diferentes métodos de secagem. Ciência Rural, Santa Maria, v. 40, n. 11, p. 2398-2401, 2010.

MARTINS, L. S. S.; MUSSER, R. S.; SOUZA, A. G.; RESENDE, L.V.; MALUF, W.R. Parasitismo de Meloidogyne enterolobii em espécies de myrtaceae. Revista Brasileira de Fruticultura, Jaboticabal, v. 35, n. 2, p. 477-484, 2013.

MENDES, A. D. R. Produção e nutrição de mudas de pessegueiro em hidroponia. 2007. 46 f. Dissertação (Mestrado em Agronomia) Universidade Federal de Lavras, Lavras, 2007.

PASQUAL, M.; CHALFUN, N. N. J.; RAMOS, J. D.; VALE, M. R do; SILVA, C. R. de R. Fruticultura comercial: propagação de plantas frutíferas. Lavras: UFLA/FAEPE, 2001. 137p.

P I C C HIONI, G.A.; WEINB A UM, S.A.; BROWN,P.H. Retention and kinetics of uptake and export of foliage-applied, labeled boron by apple, pear, prune, end sweet cherry leaves. Journal American Society Horticultural Science. Alexandria, v.120, n.1, p.28-35, 1995.
RADIN, B.; LISBOA, B. B.; WITTER, S.; BARNI, V.; JÚNIOR, C. R.; MATZENAUER, R.; FERMINO, M. H. Comportamento de cultivares e híbridos de morangueiro. Brasília: Horticultura Brasileira, Brasília, v. 8, n. 2, p. 25-36, 2011.

RODRIGUES, L. R. F. Técnicas de cultivo hidropônico e de manejo ambiental no controle de pragas, doenças e nutrição vegetal em ambiente protegido. Jaboticabal: FUNEP, 2002. 762 p.

ROZANE, D.E.; PRADO, R. M.; NATALE, W.; ROMUALDO, L. M.; SOUZA, H. A.; SILVA, S. H. M. G. M. Produção de mudas de caramboleiras B-10 e Golden Star: II - Marcha de absorção e acúmulo de nutrientes. Revista Brasileira de Fruticultura, Jaboticabal, v. 33, n. 4, p. 1308-1321, 2011.

SEIFERT, K. E.; PIO, R.; CELANT, V. M.; CHAGAS, E. A. Mudas de pera produzidas por dupla enxertia em marmeleiro utilizando o porta-enxerto 'Japonês'. Pesquisa Agropecuária Brasileira, Brasília, v. 44, n. 12, p. 1631-1635, 2009.

SOUZA, A. G. Produção de mudas enxertadas de pereira e pessegueiro em sistema Hidropônico. 2010. 91 f. Dissertação (Mestrado em Agronomia) Universidade Federal de Lavras, Lavras, 2010.

SOUZA, A. G.; CHALFUN, N. N. J.; FAQUIN, V.; SOUZA, A. A. Production of pear trees grafted under hydroponic conditions. Scientia Agraria, Londrina, v. 12 , n.1, p. 266-268, 2011 a.

SOUZA, A. G.; CHALFUN, N. N. J.; FAQUIN, V.; SOUZA, A. A. Production of peach grafts under hydroponic conditions. Ciência e Agrotecnologia, Lavras, v. 35, n.2, p. 322-326, 2011 b.

SOUZA, A. G.; FAQUIN, V.; CHALFUN, N. N. J.; SOUZA, A. A. Produção de mudas de tangerineira "Pokan" em sistema hidropônico. Ciência Agronômica, Fortaleza, v. 44, n. 4, p. 296297, 2013.

VERÍSSIMO, V.; HERTER, F. G.; RODRIGUES, A. C.; TREVISAN, R.; MARAFON, A. C. Níveis de cálcio e boro de gemas florais de pereira (pyrussp.) no sul do Brasil. Revista Brasileira de Fruticultura, Jaboticabal, v. 28, n. 1, p. 28-31, 2006. 